\title{
A Gamification-based Social Collaborative Architecture to increase resilience against natural disasters
}

\author{
Flávio E. A. Horita ${ }^{1}$, Luiz F. F. G. Assis ${ }^{1}$, Raul E. S. Castanhari ${ }^{1}$, Seiji Isotani ${ }^{1}$, \\ Wilmax M. Cruz ${ }^{1}$, João Porto de Albuquerque ${ }^{1}$ \\ ${ }^{1}$ Institute of Mathematics and Computer Science (ICMC) - University of São Paulo \\ (USP) \\ Postal Code 668 - 13.560-970 - São Carlos - SP - Brazil \\ \{horita, luizffga, raul, sisotani, wilmcruz, jporto\}@icmc.usp.br
}

\begin{abstract}
Natural disasters have led to the need for urgent measures to mitigate the effects of these accidents. The question of awareness is extremely important in dealing with disasters, and collaborative systems are very serviceable for this. In this context, the aim of this work is to outline a gamification-based social collaborative architecture (GSCA) to increase resilience against natural disasters. We have combined techniques to produce and share information through the interaction of a large number of people, such as those employed in social media and serious games. This architecture was evaluated and analyzed using the Voluntary in Action, a web platform which applies the components defined in the GSCA. The results suggest that gamification is a system with a great potential to help increase the knowledge of a community about disasters.
\end{abstract}

\section{Introduction}

The increasing occurrence of natural disasters around the world has attracted the attention of various global agencies (Mendiondo, 2010), and led to the adoption of a number of preventive and responsive measures (Baharin et al., 2009), such as giving priority to key information to allow management strategies to be employed to deal with these accidents (Bui et al., 2000), (Lu et al., 2006).

In assisting government agencies to cope with disaster management, there have been a number of volunteers who have provided valuable and updated information to community organizations and thus helped them in their recovery (Goodchild, 2007), (Gill and Bunker, 2012). However, it is difficult to manage young volunteers during a disaster because they usually act in a flexible and task-based manner in new social media, which means that the results of their participation can be seen immediately (Neubauer et al., 2013).

For this reason, collaborative systems have been employed to gather a large number of people together to carry out various tasks for several purposes, exchange experiences with each other and tackle serious problems in a distributed way (Doan et al., 2011).

This paper aims to outline an architecture to assist in the management of natural disasters. In undertaking this, common features found in collaborative and recommendation systems, social networks and geoinformatics are used for the dissemination of knowledge. These features are enhanced by gamification techniques which increase the interaction that occurs in the activities carried out by volunteers. 
The remainder of the paper is divided into four sections: Section 2 outlines the background, Section 3 describes the Gamification-based Social Collaborative Architecture (GSCA), Section 4 shows its evaluation and analysis, and Section 5 makes some final considerations and recommendations for future work.

\section{Background}

\subsection{Natural Disaster Management}

In recent years, increasing attention has been paid to environmental risks and their potential impact on natural disasters, particularly in light of the frequency with which they occur. A natural disaster is an occasion when a society is afflicted by a natural event and the damage is so great that external assistance is required (De Longueville et al., 2010).

The events in Haiti, Chile, Pakistan in 2010, Queensland between 2010-11 and Japan and Christchurch in 2011 are examples of recent disasters. Together, they accounted for 333,944 victims and economic losses estimated at U\$199-327 billion. In addition, disasters and climate change that occurred in China in 2008 caused damage of approximately U\$111 billion to its economy (Tu et al., 2009).

One way to mitigate this damage is the adoption of countermeasures to prevent a simple event from turning into a catastrophe (Baharin et al., 2009), (Poser and Dansch, 2010). Disaster management is an important alternative to help in this kind of situation (Baharin et al., 2009).

The information that is relied on for disaster management is of crucial importance and must be accurate, timely, and complete with regard to the current state of environmental variables, so that it can be used to support predictions about upcoming changes and associated impacts (Baharin et al., 2009).

However, owing to the specific needs and preferences of each person or official agency, a good deal of information related to disasters and climate change is scattered over different areas and is disconnected of poor quality (Tu et al., 2009). As a result, several issues affect the way information is created and shared, and when critical "success factors" are analyzed, there is clearly a failure to give priority to key information required for disaster management (Bui et al., 2000), (Lu et al., 2006).

\subsection{Collaborative Systems}

The growth of the web has provided some important benefits, such as the creation of an environment where teams that are scattered in geographical terms can carry out activities in a collaborative manner (Bafoutsou and Mentzas, 2002). To achieve this, collaborative computing relies on computers to support coordination and cooperation between two or more people who come together to solve a problem (Borenstein, 1992), (Schooler, 1996). The study of these systems forms part of a large area of interdisciplinary research, Computer Supported Cooperative Work (CSCW).

Commercial products are often mentioned such as CSCW groupware. According to Ellis et al. (1991), this term more commonly refers to the computer-based systems that support groups of people engaged in a common task (or goal) and that provide an interface to a shared environment". On the other hand, Schooler (1996) states that the essential feature of groupware are the creation of shared workspaces among employees. 
According to Bafoutsou and Mentzas (2002), in the literature there are several approaches that provide taxonomies and classifications of collaborative systems. These classifications and taxonomies are designed to separate these systems on the basis of their properties and characteristics.

\subsection{Crowdsourcing and Voluntereed Geographic Information (VGI)}

The emergence of Web 2.0 and evolution of mobile devices that allow content creation, have become the basis for the emergence of a new paradigm, where users in general, (i.e. citizens) become established as producers of data and information (Niko et al., 2011), which in many cases, is more detailed and of a higher quality than that provided by official agencies (Goodchild, 2007), (Elwood, 2008), (De Longueville et al., 2010), (Ostermann and Spinsati, 2011).

Heipke (2010) invented the term "crowdsourcing" for this phenomenon which involves content production being carried out by a third party, that is assigned to intelligence and knowledge. It is based on the experience of volunteers, who are independent in the way they use their free time and are located in remote and diverse areas.

In a similar context, but more closely linked to geographical issues, Goodchild (2007) used the term VGI which he defined as a collection of digital spatial data produced by individuals and informal institutions, as well as by ordinary citizens who use appropriate tools to gather and publish their observations and geographical knowledge on the web.

Some of the systems which employ these concepts are Wikimapia, OpenStreetMap, Mechanical Turk, CloudCrowd, and Wikipedia, and together they compose a set of systems defined as Crowdsourcing Systems which enlist volunteers to assist in building long- term artifacts that are beneficial to the whole community (Doan et al., 2011).

In this context, Doan et al. (2011) raised some important challenges that crowdsourcing systems have to face. First, how should these systems recruit volunteers and maintain their assistance. Second, what different kinds of support can users provide and how they can be combined. Finally, although this huge amount of data is useful to obtain knowledge, it causes several problems with regard to its quality (Elwood, 2008), (Goodchild and Li, 2012).

\subsection{Gamification}

Deterding et al. (2011) states that the term "Gamification" was coined by the digital industry. The first record of the use of this term was around 2008, but it was most widely employed in 2010. Moreover, several parallel terms that are still being used, can be found in the literature, and new terms are still being incorporated, such as: "productivity games", "surveillance entertainment", "funware", "playful design", "behavioral games", "game layer" or "applied gaming". As a result, the definition of gamification is "the use of game design elements in non-game contexts".

The identification of game elements is still a question that is much discussed, and in the literature, there are a few works that offer definitions such as "classic game model" of Juul (2005) and "Ten Ingredients of Great Games" of Reeves and Read (2009). Interpretations that are either very strict or very tolerant of these elements, can make the game either very limited or unlimited. One solution provided by Deterding et 
al. (2011) is to restrict the scope of the term, by only considering the elements that are found in most games, but not necessarily all of them.

\subsection{Related Works}

Tools and technologies based on social media concepts, have been widely employed to assist in activities and procedures related to disaster management (Pohl et al., 2012), (Horita et al., 2013). Among these, there are works whose main goal is to employ extensive data analysis techniques and information generated by Twitter, Facebook, Flickr and YouTube (Kaewkitipong et al., 2012), (Sinnappan et al., 2010), (Pohl et al., 2012), (Ahmed, 2011). There are also those based on the features that characterize crowdsourcing systems (Doan et al., 2011).

One group, in particular, stands out in the way it displays collaborative features to allow the fast creation and easy sharing of geographic content. The interactive maps created by the Ushahidi platform is one of the examples because they offer resources that can support the gathering and sharing of information with a view to solving problems emerging from an environment of uncertainty (Okolloh, 2009). Similarly, we also include platforms like Elva, Google Crisis Map, and LRA Crisis Tracker in this category. On the other hand, OpenStreetMap and Wikimapia are open technologies that provide resources for producing general street maps and georeferenced elements in a collaborative way (Haklay and Weber, 2008), (Goodchild, 2007).

In addition, there are several tasks which are hard for computer systems to execute but can be easily performed by humans, such as image recognition (Von Ahn and Dabbish, 2008). There are also technologies to recruit volunteers to conduct an analysis with the aid of aerial images taken in the affected areas like MapMill and Tomnod.

This study seeks to set out an architecture for providing resilience against natural disasters by using concepts and features of social media, collaboration, and crowdsourcing systems, all of which are combined with gamification techniques (Deterding et al., 2011) and recommender systems (Melville and Sindhwani, 2010) to increase the interaction, participation and assistance of members of communities.

\section{Gamification-based Social Collaborative Architecture (GSCA) to increase resilience to natural disasters}

According to Neubauer et al. (2013), voluntarism is the main pillar of disaster relief and occurs when people show a willingness to help communities without any financial self-interest. It should be noted that most volunteers are between 30 and 50 years old and married. This means there is a need to increase the participation of those younger than 30 and a good strategy is to offer flexible and task-based activities in new social media, where the results of their involvement can be seen in an immediate manner.

To address this question, the aim of this study is to develop a collaborative social architecture, with elements of gamification and social media components, called GSCA. This architecture is designed to create communities that are resilient to natural disasters, and hence reduce the extent of the damage that they cause. According to Pohl et al. (2012), these components can encourage the use of architecture, because people often use social media to document situations that they are involved in.

Elements of gamification, as well as social media, are coupled with GSCA with a view to encouraging volunteers to participate further (Deterding et al., 2011). For 
example, volunteers at GSCA can take part in special missions, which include the features of games like scores, badges and rankings which reflect the abilities and achievements of the participants. This architecture is based on Crowdsourcing (Doan et al., 2011) and Volunteered Geographic Information (VGI), which provide content to support the acquisition of knowledge.

According to Goodchild (2007), formerly relevant geographic information was only provided by official agencies; however, with the emergence of Web 2.0 and evolution of mobile devices, this geographic information has often come to be shared. It is known that communities have shared a good deal of information, and although individual information was often imprecise, a collective analysis of the data has often had a considerable effect.

Three specific objectives must be attained before this architecture can be implemented into a platform: (1) Providing knowledge to communities about natural disasters; (2) Mapping the reactions of communities to natural disasters; and, (3) Assisting emergency agencies in natural disaster management.

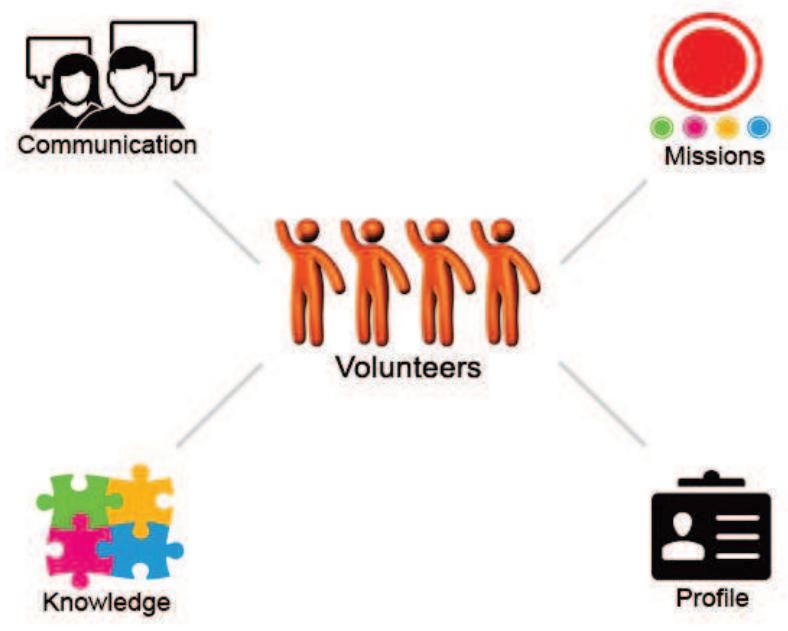

Figure 1. Components of GSCA

As can be seen in Figure 1, the volunteers are the central component of the architecture because, as shown earlier, it is they who both evaluate and increase the amount of information available and extend its range. The four other components, communication, missions, knowledge, and profile, provide mechanisms to support the central element and the volunteers, as well as enabling them to carry out their activities. The following sections outline each of these components in detail.

\subsection{Communication Component}

Communication between users occurs in three ways: (1) they can exchange messages individually or collectively, synchronously or asynchronously, (2) they can create forums to discuss ideas, which should address specific issues related to natural disaster management, and (3) they can create communities by bringing together individuals with common interests.

\subsection{User Profiles Component}

The profile of the volunteers is characterized by personal information, their degree of interest in forums and communities, and their network of volunteers (friends). Moreover, users can find out about recent activities from other users and news related to 
natural disasters.

As well as this, volunteers can participate in missions with different purposes, where they can be awarded points for their level of performance. The possession of performance skills denotes that a volunteer is able to carry out some kind of task and can thus be designated as a fireman, policeman, soldier, and so on.

A system of overall ranking is employed for all the participants, and this encourages them to become more full involved in a spirit of competitiveness (Von Ahn and Dabbish, 2008). In addition, the "missions" performed by specific users are listed chronologically in their profile to ensure more control of their activities, and this system can be applied to all the participants.

\subsection{The Missions Component}

This component of the architecture enables users to perform interactive activities while at the same time, allowing them to generate and acquire knowledge related to natural disasters. The goal of these activities is to encourage the participation of a large number of people as well as to ensure that they will continue to assist societies to become resilient to accidents such as floods or tornadoes.

This can be accomplished by offering the activities in the form of "missions", in which the participants volunteer to fulfill certain tasks with specific goals and rewards. These missions are conducted collaboratively with maps that represent real or fictitious regions, for example, forests, cities or risk areas. The architecture can also recommend specific missions for determined volunteers depending on their scores and proven skills.

\subsection{Knowledge Component}

The knowledge component is composed of three main elements: (1) news related to natural disasters, (2) exchange of experiences between volunteers and (3) missions.

News related to natural disasters serves to centralize information and keep participants updated. The exchange of experiences (related to disaster management) between participants occurs via the "Communication Component". Finally, missions help volunteers to increase their knowledge through some tasks related to natural disaster management (as described in the "Missions Component").

\section{Evaluation and Analysis}

In this section, the assessment and analysis of the outlined architecture are described in detail.

\subsection{Potential Scenario: Voluntary in Action Platform}

In a disaster situation, all the information related to the local context is important for faster and more accurate decision-making (Baharin et al., 2009). However, due to adverse structural and organizational factors, it is difficult for emergency agencies (EAs) to have proper information. According to Vivacqua and Borges (2012), available services enable communities to provide data about their region in a voluntary way. For example, an emergency scenario about the availability of active hydrants in a region, can be solved by using volunteer information.

However, this approach causes some difficulties. The quantity and quality of reported information depends on the number of volunteers willing to help with data related to their community (Goodchild and Li, 2012). Moreover, three important factors 
arise from this: (1) personal motivation; (2) professional volunteers; and (3) the possibility of creating and sharing information maliciously or for criminal purposes (Coleman et al., 2009). Finally, there is also the need to adopt mechanisms to recruit volunteers so that they can provide useful assistance to the EAs (Vivacqua and Borges, 2012).

One solution could be achieved via the integration of gamification techniques that involve practices of collaborative systems, the main objective of GSCA. Thus, a web platform called Voluntary in Action is displayed as an implementation of this architecture. Figure 2 shows an example of mapping performed on the platform mission.

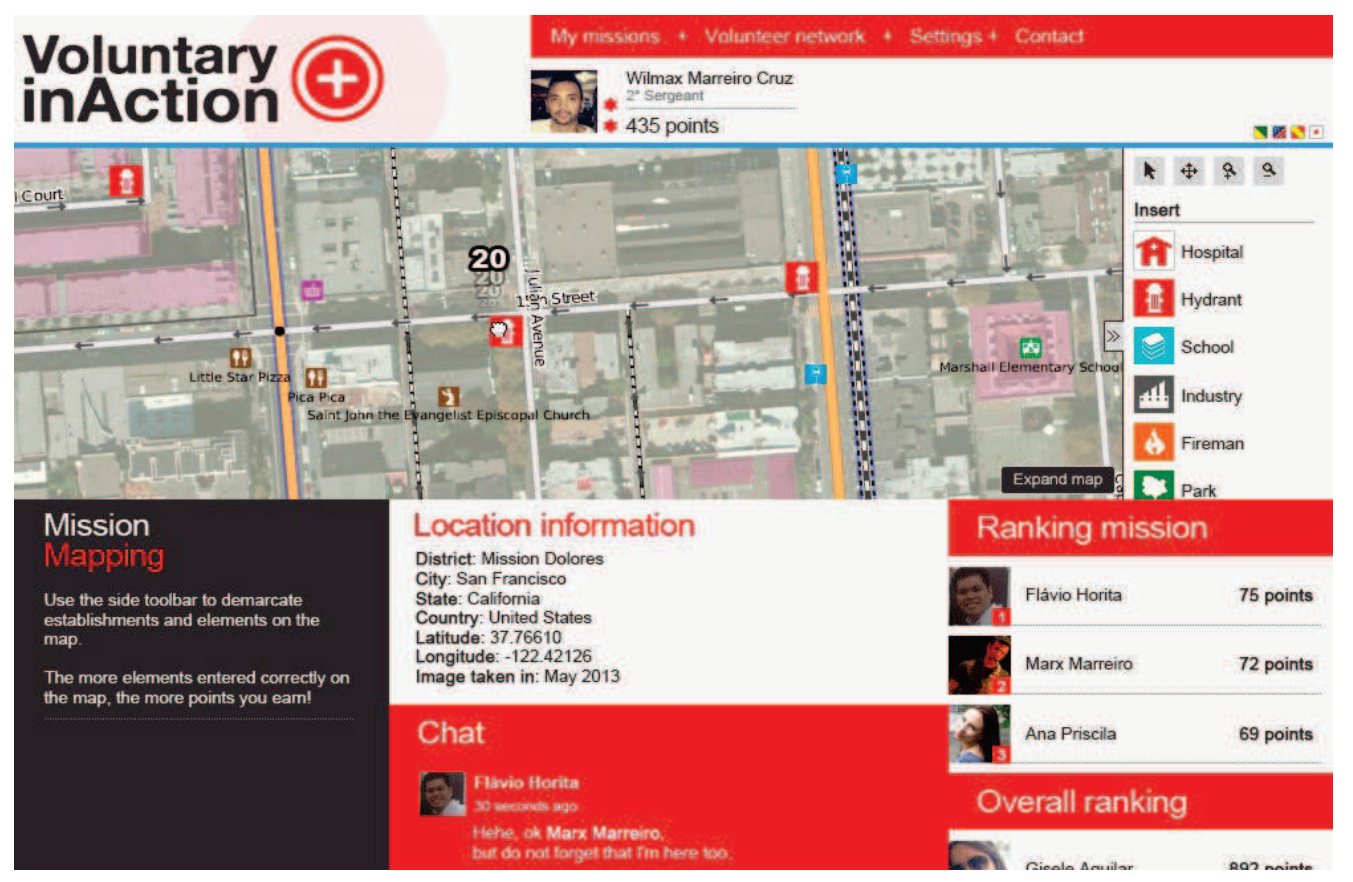

Figure 2. Components of GSCA

This mission allows the tagging of geographic features shown on a particular map. In the scenario outlined by Vivacqua and Borges (2012), this platform enables volunteers to navigate a map and by using specific components, create the reference points of existing elements in the real world, such as parks, schools and fire hydrants.

In addition, the volunteer can see the score of the volunteers of his team, his badge, overall ranking and ranking mission. These implemented elements are defined as functionalities of gamification. Thus, (as shown in Figure 2), by tagging the element, the volunteer earns some points and this allows him to improve his rankings and the merit of his badges, as well as encouraging him to compete with other volunteers. It also encourages him/her to participate in diverse activities and increase the quantity of georeferenced elements.

The platform also stores data related to how volunteers interact with each other using a chat line to achieve the mission. Through the platform, it is possible to detect factors that may influence the achievement of future actions related to disasters, and carry out missions as a basis to make recommendations and improvements.

In a complementary way, in addition to the knowledge generated in the mapping mission, there is also the possibility of forming data through image analysis, which is 
conducted by volunteers or by shared web tools such as Google Street View. This means that Figure 3 has another mission platform, (georeferencing), which carries out an analysis of random images to define locations where there is evidence of useful elements in disaster management.

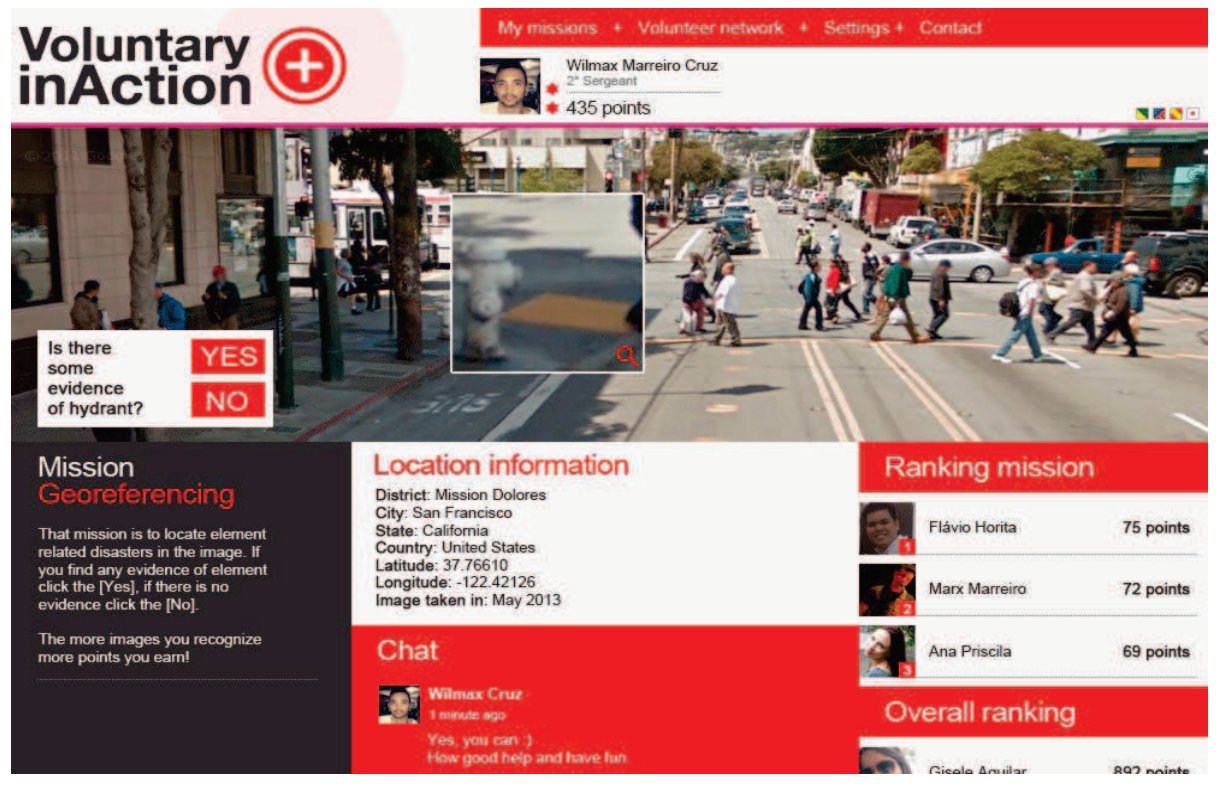

Figure 3. Georeferencing Mission

As can be seen in Figure 3, in this mission a random image is displayed, and includes a magnifying glass feature, which aims to answer some of the available questions shown on the left-hand side. In the context described by Vivacqua and Borges (2012), this question was linked to the presence or absence of fire hydrants. The gamification resources, such as rankings and scores for created elements are employed to encourage volunteers to get involved.

Figure 4 depicts the platform homepage, where users can view the scope of the project through the possible missions that they can select from the menu options of the platform, and obtain some news related to natural disasters.

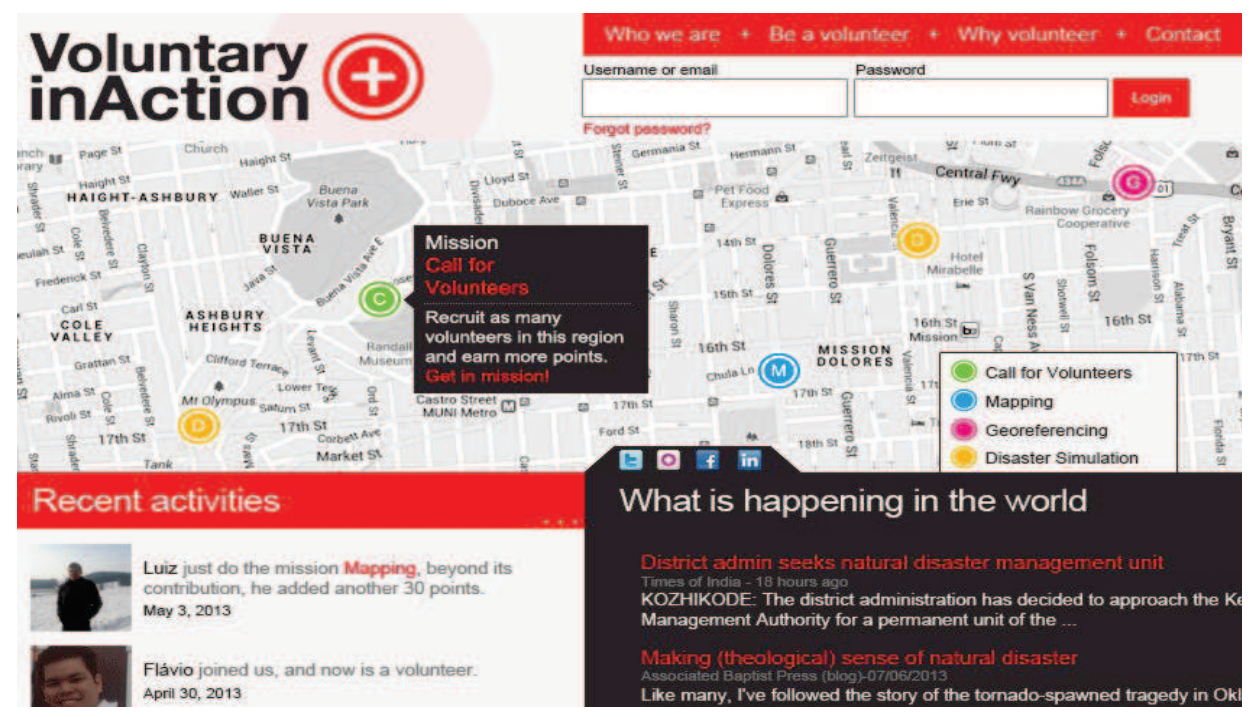

Figure 4. Platform Homepage 
Finally, Figure 5 displays a user profile, which contains his personal information, his performed missions in chronological order (timeline), his friends and communities, as well as his overall ranking and some missions that have been recommended to him.

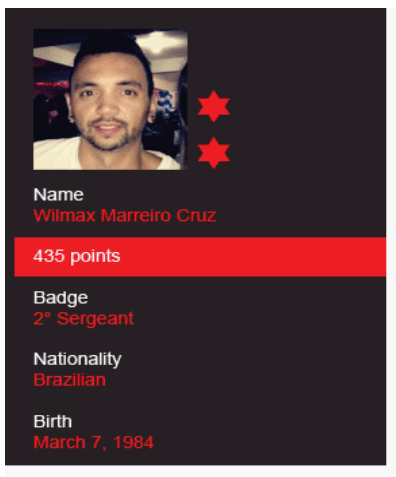

Friends . 32

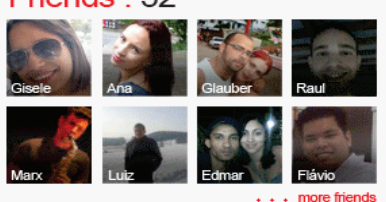

Communities . 7
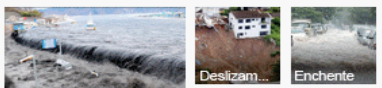

Missions (timeline) . 15

May 2013
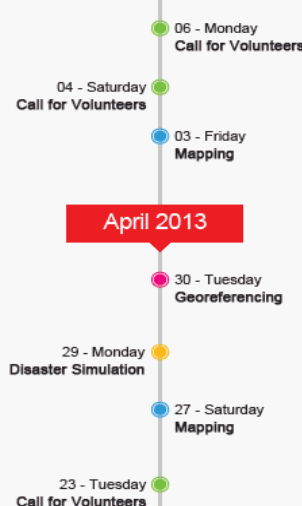

Call for Volunteers

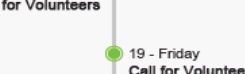

18- Wednesday

19 - Friday
Call for Volunte

Georeferencing

\section{Overall ranking}

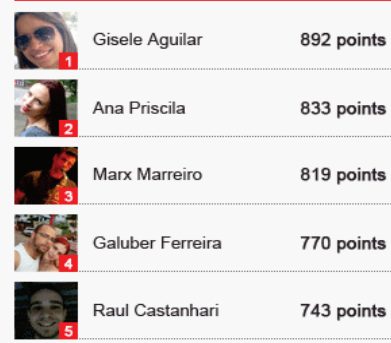

Missions recommended

(C) Call for Volunteers

(G) Meoreferencing $_{2,2013}$

(M) Mapping

(C) Call for Volunteers

Figure 5. User Profile

A timeline's mission of a user is an important element in the user profile. The timeline can help the user to manage his activities on the basis of his performed missions, and in addition, it can encourage other participants to carry out certain tasks. Moreover, this platform can also suggest missions to a user by drawing on his personal information and his performance in old missions.

\section{Final Considerations}

The damage caused by natural disasters has encouraged the development of systems that support the management of such events. Many of these attempts aim at providing considerable assistance to the general population and, more specifically, to people which live in risk areas. In this manner, the use of collaborative techniques has proved to be effective for solving certain complex tasks.

People can overcome traditional models of data analysis and machine learning when they work on tasks that are simple for human beings but difficult for computers and, moreover, they can achieve great results in a much shorter period of time compared with the solutions provided by more traditional techniques. The use of collaborative techniques (including gamification techniques which are used to maximize its results) provides an innovative alternative and can be used in various scenarios.

In this context, the effective participation of people can lead to communities who are resilient to natural disasters. In this paper, we have outlined a gamification-based social collaborative architecture which is designed to work in this scenario. Moreover, the information it provided for its application in the Voluntary in Action platform, shows the benefits of increasing the amount of information since this can be useful in assisting the activities of the emergency agencies in their preventive and reactive work. 
This architecture can lead to the generation, acquisition and dissemination of necessary knowledge and thus reduce the frequency and intensity of natural disasters, by fostering health and safety awareness among people who live in a risk area.

In future work, the platform could provide specific features for professionals of emergency agencies, will be added. In this way, they can create their own missions to increase the knowledge generated by the platform, and thus eliminate the problem of a possible lack of information. Furthermore, there will be a chance to locate professionals who are working in risk areas through maps processed in real time, and thus aid the coordination of field activities.

\section{References}

Ahmed, A. (2011). Use of social media in disaster management. In ICIS 2011Proceedings.

Bafoutsou, G., and Mentzas, G. (2002). Review and functional classification of collaborative systems. International Journal of Information Management 22, 4, 281 305.

Baharin, S., Shibghatullah, A., and Othman, Z. (2009). Disaster management in Malaysia: An application framework of integrated routing application for emergency response management system. In International Conference of Soft Computing and Pattern Recognition (SOCPAR), pp. $716-719$.

Balabanovic, M., and Shoham, Y. (1997). Fab: content-based, collaborative recommendation. Commun. ACM 40, 3, pp. 66-72.

Borenstein, N.S. (1992). Computational mail as network infrastructure for computer-supported cooperative work. In Proceedings of the 1992 ACM conference on Computer-supported cooperative work, CSCW'92, ACM, pp. 67-74.

Bui, T., Cho, S., Sankaran, S., and Sovereign, M. (2000). A framework for designing a global information network for multinational humanitarian assistance/disaster relief. Information Systems Frontiers 1, 4, 427-442.

Coleman, D. J., Georgiadou, Y., Labonte, J., Observation, E., and Canada, N. R. (2009). Volunteered Geographic Information : the nature and motivation of producers. International Journal of Spatial Data Infrastructures.

De Longueville, B., Luraschi, G., Smits, P., Peedell, S., and De Groeve, T. (2010). Citezens as sensors for natural hazards: a VGI integration workflow. Geomatica 1.

Deterding, S., Dixon, D., Khaled, R., and Nacke, L. (2011). From game design elements to gamefulness: defining "gamification". In Proceedings of the 15th International Academic MindTrek Conference: Envisioning Future Media Environments (New York, NY, USA, 2011), ACM, pp. 9-15.

Doan, A., Ramakrishnan, R., and Halevy, A. Y. (2011). Crowdsourcing systems on the world-wide web. Commun. ACM 54, 4, 86-96.

Ellis, C. A., Gibbs, S. J., and Rein, G. (1991). Groupware: some issues and experiences. Commun. ACM 34, 1, 39-58.

Elwood, S. (2008). Volunteered geographic information: future research directions motivated by critical, participatory, and feminist GIS. GeoJournal 72, 3-4, 173-183. 
Goodchild, M. F. (2007). Citizens as sensors: the world of volunteered geography. In Geojournal, pp. 1-12.

Goodchild, M. F., and Li, L. (2012). Assuring the quality of volunteered geographic information. Spatial Statistics 1, 0, 110-120.

Haklay, M., and Weber, P. (2008). Openstreetmap: User-generated street maps. Pervasive Computing, IEEE 7, 4, 12-18.

Heipke, C. (2010). Crowdsourcing geospatial data. ISPRS Journal of Photogrammetry and Remote Sensing 65, 6, 550-557.

Horita, F. E. A., Degrossi, L. C., de Assis, L. F. G., Zipf, A., and de Albuquerque, J. P. (2013). The use of volunteered geographic information (VGI) and crowdsourcing in disaster management: a systematic literature review. In 19th Americas Conference on Information Systems (AMCIS).

Juul, J. (2005). Half-Real: Video Games between Real Rules and Fictional Worlds. The MIT Press.

Kaewkitipong, L., Chen, C., and Ractham, P. (2012). Lessons learned from the use of social media in combating a crisis: A case study of 2011 Thailand flooding disaster. In Thirty Third International Conference on Information Systems.

Lu, D.-K., Pettit, S., and Beresford, A. (2006). Critical success factors for emergency relief logistics. WHAMPOA - An Interdisciplinary Journal 51, pp. 177-184.

Mendiondo, E. M. (2010). Reducing vulnerability ti water-related disasters in urban areas of the humid tropics. In Integrated Urban Water Management Humid Tropics, Paris, France, pp. 109-127.

Melville, P., and Sindhwani, V. (2010). Encyclopedia of Machine Learning. Springer, ch. Recommender Systems, pp. 829-838.

Niko, D. L., Hwang, H., Lee, Y., and Kim, C. (2011). Integrating user-generated content and spatial data into web gis for disaster history. Computers,Networks, Systems, and Industrial Engineering 2011.

Neubauer, G., Nowak, A., Jager, B., Kloyber, C., Flachberger, C., Foitik, G., and Schimak, G. (2013). Crowdtasking - A New Concept for Volunteer Management in Disaster Relief. Internation Symposium on Environmental Software Systems, 345-356.

Okolloh, O. (2009). Ushahidi or "testimony": Web 2.0 tools for crowdsourcing crisis information. Participatory Learning and Action 59, 65-70.

Ostermann, F. O., and Spinsanti, L. (2011). A Conceptual Workflow For Automatically Assessing The Quality Of Volunteered Geographic Information For Crisis Management. In The 14th AGILE International Conference on Geographic Information Science.

Pohl, D., Bouchachia, A., and Hellwagner, H. (2012). Supporting crisis management via sub-event detection in social networks. In 2012 IEEE 21st International Workshop on Enabling Technologies: Infrastructure for Collaborative Enterprises (WETICE).

Poser, K., and Dransch, D. (2010). Volunteered geographic information for disaster management with application to rapid flood damage estimation. Geomatica 64, 1, pp. 89-98. 
Reeves, B., and Read, J. L. (2009). Total Engagement: Using Games and Virtual Worlds to Change the Way People Work and Businesses Compete. Harvard Business School Press.

Schooler, E. Conferencing and collaborative computing. Multimedia Systems 4 (1996), pp. 210-225.

Sinnappan, S., Farrell, C., and Stewart, E. (2010). Priceless tweets! a study on twitter messages posted during crisis: Black Saturday. In ACIS 2010 Proceedings.

Tu, Y., Li, Q., and Liu, R. (2009). A geospatial information portal for emergency management of natural disasters. In 2009 IEEE International Geoscience and Remote Sensing Symposium (IGARSS), vol. 2, pp. 404-407.

Vivacqua, A. S., and Borges, M. R. (2012). Taking advantage of collective knowledge in emergency response systems. Journal of Network and Computer Applications 35, $1,189-198$.

Von Ahn, L., and Dabbish, L. (2008). Designing games with a purpose. Commun. ACM 51,8, pp.58-67. 